\title{
La evaluación de la transparencia y la apertura de las universidades en España y América Latina. Estudio de caso de la Universidad de Buenos Aires.
}

\author{
The evaluation of transparency and openness at universities in Spain and Latin \\ Amercia. Case study of University of Buenos Aires.
}

\section{AUTOR 1: \\ Dr. José Luis Ros-Medina}

Licenciado en Ciencias Políticas con Premio Extraordinario, Máster en Gobierno, Administración y Políticas Públicas, y en Técnicas de Investigación Social. Investigador predoctoral en la U. de Murcia, Coordinador Nacional de RAGA España e investigador NovaGob y Cátedra RSC UM.ORCID: 0000-00032903-6632.

Correo: joseluis.ros1@um.es

\section{AUTOR 2:}

Alejandro Mañogil Ros Graduado en Ciencias Políticas y Gestión Pública y estudiante de Máster en Análisis Político Aplicado en la Universidad de Murcia. Gestor de proyectos e investigador junior en NovaGob y de la Red InnoLabs.

Coordinador administrativo de RAGA España.ORCID:

\section{Correo:}

alejandro.manogilr@um.es
Recibido: 21 de agosto de 2021

Aprobado: 16 de noviembre 2021

\section{RESUMEN:}

Desde hace 10 años la Fundación Compromiso y Transparencia lleva publicando el que es el índice de evaluación de la transparencia y apertura universitaria más completo, exigente y referenciado en España. Su último informe publicado somete a examen a 75 universidades y presenta como datos más relevantes que la transparencia universitaria en España es la más alta desde el inicio de la serie y que las fallas en transparencia vienen dadas sobre todo por la falta de actualización. Sin embargo, en América Latina, las experiencias evaluativas sobre transparencia universitaria han sido mínimas. Se da, por tanto, un ámbito del conocimiento público por explorar y oportuno en el contexto tan complejo y exigente de la era post Covid19, donde deberíamos enfocar nuestros esfuerzos en tener instituciones públicas más abiertas y transparentes, siendo las universidades una de las palancas más importantes para el cambio. Así, este trabajo reflexiona sobre la evaluación de la transparencia universitaria, realiza una propuesta de adaptación para América Latina y un estudio de caso de aplicación de la misma para la Universidad de Buenos Aires, como propuesta para extender a un marco regional latinoamericano la evaluación de la transparencia universitaria mediante un índice anual y su ranking correspondiente.

Palabras clave: transparencia, evaluación, Índice de Transparencia Universitaria de Latinoamérica, INTULA, Universidad de Buenos Aires, Fundación Compromiso y Transparencia

\section{ABSTRACT:}

Over the last 10 years, the Compromise and Transparency Foundation has been publishing the most detailed, demanding and referenced index of assessment for transparency and openness at universities in Spain. Its latest published report examines 75 universities and shows as the most relevant data that transparency in the running of universities in Spain is the highest since the start of the series and that the failures regarding transparency are mainly due to the lack of updating.

However, the assessment reports on transparency carried out in Latin America Universities have been very few. Therefore, there is an area of public knowledge to be explored which is also very appropriate in the complex and demanding context of the post Covid-19 era, where we should focus our efforts on having more open and transparent public institutions. Bearing in mind that universities are one of the most important keys leading to change. In this way, this report reflects on the assessment of transparency in universities, it develops an adaptation proposal for Latin America as well as a specific application case of study for the University of Buenos Aires as a suggestion to extend to a Latin-American regional framework the assessment of transparency in the running of universities using an annual index and its appropriate ranking.

Keywords: transparency, evaluation, Index of Transparency at Universities in Latin America, University of Buenos Aires, Compromise and Transparency Foundation. 


\section{INTRODUCCIÓN}

\section{La evaluación de la transparencia activa}

La transparencia es una de las cualidades de la administración pública que más ampliamente se ha defendido en las últimas décadas por parte de la Academia, tanto como fin en sí misma como medio para lograr otras cualidades consideradas generalmente positivas en la Administración Pública, como la ausencia de corrupción, la eficiencia o la rendición de cuentas. También la opinión pública ha considerado la transparencia como una de las cualidades más exigibles a los poderes públicos y, no en vano, se han generado cientos de proyectos desde la sociedad civil a escala nacional e internacional en todo el mundo. Actores como Transparency International (TI) son ya parte del mapa cotidiano de la Administración Pública. En esta línea, y casi siempre de la mano de proyectos de la sociedad civil que han pretendido ejercer como controladores externos de la acción pública, se han generado acciones concretas de evaluación, monitoreo y seguimiento. Uno de los más notables ha sido el de la lucha contra la corrupción, donde el Índice de Percepción de la Corrupción de TI lleva desde 1995 evaluando la evolución de la corrupción a nivel nacional, habiendo evaluado en su última edición publicada, la de 2020, a un total de 179 países. Otros proyectos han ampliado el marco de análisis introduciendo la propagación de la corrupción como variable clave; un ejemplo sería el Índice de Gobernanza del Banco Mundial, surgido en 1996 (Kaufmann et al., 1999), o mucho más recientemente, el Índice Europeo de Calidad de Gobierno desarrollado desde 2010 por el Instituto de Calidad de Gobierno de la Universidad de Gotemburgo (Rothstein y Teorell, 2008).

Estos proyectos evaluativos de la calidad e integridad institucional, así como su ausencia de corrupción, que sería una parte de la primera, se han convertido a lo largo de los años en herramientas básicas para el estudio que las Ciencias Sociales, especialmente la Ciencia Política, realizan del devenir de lo público. Han significado un termómetro imparcial, independiente y confiable para evaluar en qué medida las instituciones públicas progresan o retroceden en una escala de integridad. Vemos cómo, a lo largo del tiempo y sucesivamente en los distintos indicadores -que son muchos más que los tres citados a modo de ejemplo por ser de especial relevancia-, hay algunos países que registran valoraciones deficientes y regresivas y cómo otros registran valoraciones muy positivas e, incluso, al alza. Vemos cómo, además, estos índices nos revelan trayectorias de dependencia institucional muy fuertes, haciendo que el mundo de la integridad no se parezca nada al vinícola, en el que una buena añada destaca 
sobre el resto y una mala puede echar por tierra el trabajo de otras muchas. Las trayectorias institucionales suelen ser lentas, salvo en los casos de retrocesos abruptos fruto de regímenes dictatoriales o populistas que producen giros radicales hacia lo que se conoce como democracias delegativas (O'Donnell, 1994).

Dentro de la misma familia de índices destinados a evaluar los diferentes subconceptos de la integridad institucional, encontramos una familia orientada a la evaluación y el ranqueo de la transparencia activa de distintos conjuntos institucionales, ya sean públicos o privados. España tiene una larga y positiva tradición en este sentido. Un país que fue de los últimos de Europa en tener una ley de libertad de información, de hecho, fue el último de este continente en tenerla de los que superan el millón de habitantes, hizo de la necesidad virtud y generó una suerte de proyectos de control ciudadano en esta área. Así, cuando la Ley de Transparencia, Acceso a la Información y Buen Gobierno vio la luz, el 9 de diciembre de 2013, organizaciones como Transparencia Internacional España, ya habían realizado una labor ingente de evaluación y monitoreo de la transparencia en conjuntos institucionales como los ayuntamientos de mayor población (en 2008, 2009, 2010 y 2012), todas las Comunidades Autónomas (en 2010 y 2012), de todas las diputaciones provinciales (en 2012 y 2013) y de los organismos de gestión de cuencas hídricas (en 2010, 2012 y 2013). Es decir, sólo Transparencia Internacional España ya había desarrollado un total de once oleadas evaluativas en cuatro conjuntos de instituciones públicas, antes siquiera de que España tuviera una normativa nacional de transparencia.

Transparencia Internacional España ha generado, además, una gran escuela de evaluación de la transparencia en nuestro país. Antes de la aprobación de la Ley 19/2013, le siguieron en este camino la Fundación Lealtad para la verificación de la transparencia y la honestidad de las ONG o el Mapa Infoparticip@ surgido en la Universidad Autónoma de Cataluña en 2012 (aunque el grueso de su acción evaluativa lo ha desarrollado a partir de 2013). Pero el caso de mayor impacto en la evaluación de la transparencia más allá de los rankings de TI, ha sido y sigue siendo, el de la Fundación Compromiso y Transparencia (anteriormente conocida como Fundación Compromiso Empresarial). Esta fundación comenzó en 2009 a publicar su ranking de transparencia y buen gobierno de las fundaciones españolas, en 2010 el ranking de transparencia de los medios de comunicación y el ranking de transparencia de los museos, en 2012 el ranking de transparencia de los partidos políticos y transparencia de las universidades, en 2013 el ranking de transparencia de las empresas que cotizan en el índice bursátil IBEX 35, en 2017 su ranking de transparencia sobre el compromiso social y ético de las empresas farmacéuticas, en 2018 su ranking sobre transparencia 
editorial y, por último hasta ahora, en 2019 lanzan su ranking sobre transparencia de las diócesis españolas.

Tabla 1. Relación de ránkings realizados por la Fundación Compromiso y Transparencia

\begin{tabular}{|c|c|c|c|}
\hline Sector & N.․ Ediciones & 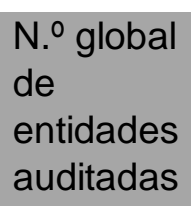 & 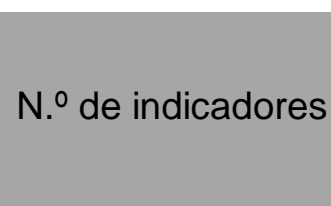 \\
\hline Diócesis & 1 & 67 & 18 \\
\hline Farmacéuticas & 1 & 26 & 13 \\
\hline $\begin{array}{l}\text { Auditoría externa de las } \\
\text { aseguradoras }\end{array}$ & 2 & 96 & Entre 5 y 6 \\
\hline Partidos Políticos & 7 & 139 & Entre 25 y 28 \\
\hline Fundaciones Políticas & 6 & 231 & 15 \\
\hline Fundaciones & 10 & 837 & Entre 13 y 20 \\
\hline Transparencia Fiscal del Ibex 35 & 7 & 245 & Entre 12 y 13 \\
\hline $\begin{array}{l}\text { Transparencia Fiscal de } \\
\text { Multinacionales }\end{array}$ & 3 & 105 & 5 \\
\hline $\begin{array}{l}\text { Gestión del talento sénior en el lbex } \\
35\end{array}$ & 3 & 105 & Entre 14 y 17 \\
\hline Auditoría externa del Ibex 35 & 3 & 105 & Entre 7 y 9 \\
\hline $\begin{array}{l}\text { Independencia y credibilidad de los } \\
\text { Medios de Comunicación }\end{array}$ & 3 & 62 & 19 \\
\hline $\begin{array}{l}\text { Emisión de contenidos de los Medios } \\
\text { de Comunicación }\end{array}$ & 3 & 28 & Entre 17 y 21 \\
\hline Museos & 5 & 291 & Entre 14 y 25 \\
\hline Universidades & 9 & 603 & Entre 25 y 27 \\
\hline
\end{tabular}

Fuente: elaboración propia a partir de la web de la Fundación Compromiso y Transparencia.

\section{La evaluación de la transparencia de las universidades}

Las universidades públicas y privadas son instituciones nucleares de las sociedades modernas y ameritan para seguir siendo parte esencial de los cambios que estas protagonizan y protagonizarán en el futuro. Por ello, no son ajenas a las complejas y siempre activas transformaciones que estamos viviendo desde hace décadas y, especialmente, desde el advenimiento de la era tras la aparición de la Covid-19. Hoy en día, las universidades de todo el mundo enfrentan cambios disruptivos que pasan por lugares comunes como la transformación digital, la ruptura desde un modelo de títulos a un modelo de competencias o habilidades, la irrupción de miles de nuevos centros de 
estudios privados basados en la formación online y la transformación a lo online en todos los centros existentes, el paso de la solidez de décadas pasadas a la liquidez en los empleos y, por tanto, en la formación, etc. Ante este complejo panorama, las universidades no pueden ser silos estancos de conocimiento, cerrados, desconectados, oscurecidos; en la sociedad red en la que todo está conectado, las universidades deben ser una palanca de cambio social y situarse a la vanguardia del nuevo paradigma tras la Covid-19.

Por ello, la apertura y transparencia universitaria, su ultra-interconexión social, la participación de todos sus miembros y de agentes externos en la decisión de sus currículos, sus estrategias y sus decisiones cotidianas, es una absoluta necesidad. Para participar realmente de esta nueva normalidad y seguir siendo actores fundamentales, las universidades deben ser altamente exigentes con su organización interna, la cual debe pasar por una publicación activa de sus contenidos fundamentales para que tanto sus miembros (alumnos, profesores e investigadores y personal burocrático) como el resto de la sociedad, conozcan las decisiones y justificaciones de las mismas, los costos y los resultados. Asimismo, también deben adoptar modelos de mayor apertura y calidad participativa y porque estos contenidos sean parte importante y transversal de sus currículos académicos. No sólo deben practicar la apertura y la participación, sino también inspirarla y enseñarla.

Sin embargo, las experiencias evaluativas de la transparencia son mucho menos frecuentes en América Latina que en España. Concretamente, la evaluación de la transparencia de las universidades, ha tenido en el contexto de los países de Latinoamérica una aplicación mínima, no existiendo ningún índice asentado y sostenido en el tiempo, ni tampoco ninguno que haya realizado comparaciones entre universidades de toda la región. Por ejemplo, podemos citar entre los casos de aplicación de una estrategia evaluativa de la transparencia de las universidades, una investigación que evaluó la transparencia de 84 universidades en Colombia cuyo trabajo de campo se realizó durante 2015 y que tuvo en cuenta un total de 92 indicadores (Cousido et al., 2017). De estos 92 indicadores, 56 estaban orientados a evaluar la transparencia formal, la publicación de informaciones consideradas idóneas para la transparencia, así como 36 orientados a evaluar la transparencia sustantiva, la publicación de informaciones exigidas por la Ley 1712/2014. Este estudio reveló importantes conclusiones sobre el mapa de transparencia de Colombia: en primer lugar, que el nivel medio global de transparencia era bajo, con 38,6 puntos sobre 100 , en segundo, que claramente se da mayor transparencia en las universidades públicas que en las privadas, en tercero, que la transparencia formal era más altamente cumplida que la transparencia sustantiva en torno a la Ley 1712/2014, y, en cuarto y último, que la 
mayoría de las diez universidades más transparentes según este estudio no estaban acreditadas como instituciones educativas de Alta Calidad por el Ministerio de Educación Nacional de Colombia, lo que probablemente nos indique la falta de relevancia que este organismo confiere a la transparencia de las universidades.

En ese mismo año, en 2015, pero esta vez en Brasil, podemos destacar otra investigación que aborda la transparencia de las universidades federales del país (Zorzal, 2015). En este caso, la autora selecciona cinco de las 63 universidades federales brasileñas, una por cada región geográfica del país utilizando como criterio el Ranking de Webometrics de julio de 2013 para escoger aquella institución con el puesto más elevado de cada una de esas cinco regiones. La investigación mide catorce variables divididas en 110 indicadores con dos categorías dicotómicas cada uno en función de si la institución cumple o no con ese indicador. Las catorce variables son: certificaciones externas e internas; evaluación de desempeño y comparabilidad; comprensibilidad; control; divulgación; gobernanza; política contable; riesgos; oportunidades; aspectos operativos y estratégicos; información ambiental; información social; gestión del patrimonio y relaciones con la sociedad. En función del cumplimiento de los indicadores, se crean cinco niveles de transparencia (bajo, medio-bajo, medio, medio-alto y alto) y se concluye que ninguna de las 5 universidades analizadas se encuentra en el nivel alto. Se encuentran tres en el nivel medio-alto y dos en el medio (Zorzal, 2015).

También podemos citar entre estos casos relevantes el publicado en 2018 sobre las universidades de Centroamérica, incluyendo a centros universitarios de Costa Rica, Guatemala, El Salvador, Honduras, Nicaragua y Panamá (Parra Flórez, 2018). Este estudio sometió a evaluación un total de 31 universidades, las únicas de los países seleccionados que se encontraban por encima del tipo 300 QS para América Latina del año 2015. El estudio sometió a evaluación 31 indicadores dirigidos a la transparencia de la información institucional, de estructura y organización, financiera, de liderazgo y acuerdos estratégicos, y, finalmente, sobre la presentación y accesibilidad de la información (que se incluye como un quinto bloque, aunque no se debería considerar como un bloque más, sino como una exigencia transversal al resto). Los resultados más relevantes de este estudio son: la información institucional es la más ampliamente publicada mientras que el resto de ítems se cumplen mucho menos, destacando la información financiera y estratégica como las menos publicadas; todos los conjuntos nacionales suspenden caminando desde el 44,15\% de cumplimiento de las universidades de El Salvador, hasta el $30,54 \%$ de las de Guatemala. 
Otros estudios recientes se han centrado sólo en la transparencia de las universidades privadas, dentro de la corriente de la administración de empresas. Por ejemplo, en 2019 se publicó un estudio destinado a este universo que evaluó a 76 universidades privadas de Perú (Mamani Bravo et al., 2019). Este estudio tuvo en cuenta 42 indicadores destinados a evaluar la transparencia sobre el gobierno corporativo, la información para los estudiantes, la plana docente, la satisfacción y los reconocimientos, la Investigación, las titulaciones y convalidaciones, la información financiera y, por último, sobre el bienestar y la responsabilidad social. Los resultados más relevantes de este estudio fueron que el $21 \%$ de las universidades evaluadas se consideraron "transparentes" al cumplir de 36 a 42 de los indicadores, el 55\% se consideraron "translúcidas" al cumplir sólo de 25 a 35 y, por último, un $24 \%$ se consideraron "opacas" al cumplir menos de 25 indicadores; de hecho, una universidad, la Universidad Privada Telesup cumplió sólo 3 de los 42 indicadores, lo que vendría a suponer una opacidad casi absoluta. Además, y al revés que sucedía en el estudio de Cousido et al. (2017), la acreditación de la Superintendencia Nacional de Educación Superior Universitaria (SUNEDU) como "licenciadas" sí suponía un incentivo muy importante a la transparencia, por cuanto las que pertenecían a este grupo eran un $39 \%$ transparentes y un $11 \%$ opacas, mientras que las no licenciadas sólo eran en un $10 \%$ transparentes y en un $31 \%$ opacas.

Ante este panorama académico y de la sociedad civil, se hace más necesario un nuevo impulso a la evaluación de la transparencia universitaria en Latinoamérica. En esta línea, los índices de evaluación de la transparencia de las universidades, se pueden convertir, como ya lo han hecho en España, en un motor de transformación y apertura de las universidades latinoamericanas. Creemos que se debe implementar un índice de evaluación de estas instituciones que se aplique año tras año de forma sostenible a las universidades más importantes del continente con el objetivo de servir de acicate para su transformación organizativa interna y hacia el exterior, algo que se antoja imprescindible máxime en una región donde la pobreza y la desigualdad imponen retos incluso mayores que en el resto del mundo en esta era post-covid. Para ello, consideramos que un índice que sea realmente efectivo en esta misión debe cumplir unas características elementales de validez, calidad y fiabilidad, que, bajo nuestro criterio deben ser: 1) evaluar el cumplimiento de datos e información realmente relevante para que tanto la comunidad universitaria como el resto de la sociedad puedan juzgar las acciones de estas instituciones; 2) que dicha información esté orientada no sólo a la transparencia sino a la comprensión y a la rendición de cuentas; 3) que se evalúe no sólo la cantidad de información publicada sino también su calidad, condición necesaria para una efectiva comprensión y rendición de cuentas; 4) que la configuración de este 
índice no permita el cumplimiento de baja calidad o translúcido, es decir, ocultar la información más sustantiva con la obtención de notas positivas o, incluso, altas; 5) que los indicadores sean evaluados de forma gradual, de manera que se contemple el cumplimiento parcial para dotarlos de mayor precisión; y, por último, 6) que los índices se puedan aplicar a contextos normativos diferentes, como es el caso de los marcos universitarios de los distintos países de Latinoamérica.

\section{La metodología de evaluación de la transparencia de las universidades de la Fundación Compromiso y Transparencia}

La última evaluación publicada por esta fundación sobre la transparencia voluntaria en las webs de las universidades españolas es la correspondiente al año 2019, siendo la novena desde 2010. En esta última edición, se evaluaron 49 universidades públicas y 26 privadas (siendo la muestra global de 75 instituciones), y presenta como datos más relevantes que la transparencia universitaria en términos globales en España es la más alta desde el inicio de la serie, que las universidades públicas son claramente más transparentes que las privadas y que las fallas en transparencia vienen dadas sobre todo por la falta de actualización de la información publicada (Barrio et al., 2020). El índice ha evolucionado mucho en sus nueve ediciones, desde la primera en la que se evaluaron 50 universidades, y que presentó como principales conclusiones la asimetría en la transparencia de la información, desde el 100\% de publicación de la oferta universitaria, hasta un $0 \%$ de publicación de la demanda de plazas, también porcentajes muy bajos en indicadores y áreas clave como el 12\% de publicación del claustro de profesores o una generalizada carencia en transparencia de los resultados y de la información financiera (Barrio y Martin Cavanna, 2012). En la Tabla 2 podemos comprobar que el índice se ha mantenido estable en la mayoría de ediciones en torno a las 75 universidades auditadas y evolucionando de 25 a 27 indicadores.

Tabla 2. Relación de ránkings de transparencia universitaria en España realizados por la Fundación Compromiso y Transparencia

\begin{tabular}{lll} 
Año & Muestra & Indicadores \\
\hline 2019 & 75 & 27 \\
\hline 2018 & 75 & 27 \\
\hline 2017 & 26 & 27 \\
\hline 2016 & 75 & 27 \\
\hline
\end{tabular}




\begin{tabular}{lll}
2015 & 75 & 26 \\
\hline 2014 & 75 & 26 \\
\hline 2013 & 76 & 26 \\
\hline 2012 & 76 & 25 \\
\hline 2011 & 50 & 25
\end{tabular}

Fuente: elaboración propia a partir de la web de la Fundación Compromiso y Transparencia.

Los criterios evaluativos por los que se ha regido la fundación para realizar este informe han evolucionado a lo largo del tiempo. Si bien en su primera edición se centraron en: 1) la visibilidad de la información, es decir, que el lugar de publicación de la información en la web sea claramente visible, 2) la accesibilidad web, que hace referencia a que la estructura web cumpla con la Iniciativa de Accesibilidad Web (WAI), 3) la actualización, exigiendo que la información se refiera al último ejercicio cerrado legalmente, y 4) la comprensibilidad, que exige que la información publicada esté al menos en castellano (Barrio y Martin Cavanna, 2012). En cambio, en su última edición, los criterios evaluativos evolucionaron manteniéndose a grandes rasgos iguales los tres primeros, aunque el criterio de accesibilidad ya no hace referencia a la Iniciativa de Accesibilidad Web, sino solo a que no sean necesarios permisos o registros para consultar la información, mientras que el último criterio, el de la comprensibilidad, ya no se emplea y en su lugar aparece el criterio de integridad, que bajo nuestro punto de vista es mucho más necesario, siendo éste el cumplimiento de una publicación completa y exhaustiva de la información (Barrio et al., 2020).

También han evolucionado los indicadores empleados de la primera a la última edición del estudio, pasando de 25 a 26. Fundamentalmente lo que ha sucedido es que se han ido introduciendo nuevos indicadores más exigentes a costa de fusionar otros y de eliminar alguno que era de fácil y generalizado cumplimiento. Los principales cambios respecto de la primera edición han sido: la misión y el plan estratégico que en la primera edición eran dos indicadores diferentes, se han fusionado en uno sólo; la oferta de títulos o el número de alumnos matriculados en cada título, se han eliminado; y, por último, la obligatoriedad de publicar gastos e ingresos desglosados que eran dos indicadores diferentes de la publicación del presupuesto aprobado, se han introducido en este último con la exigencia de que se publique el presupuesto "detallado" (Barrio et al., 2020; Barrio y Martin Cavanna, 2012). 
A costa de este nuevo espacio generado se han introducido nuevos indicadores: la publicación de las fechas de reuniones de los órganos de gobierno y los acuerdos adoptados en las mismas; se genera un apartado nuevo de indicadores dirigidos al Consejo Social con tres indicadores sobre dotación presupuestaria y estructura organizativa, un informe de responsabilidad social con un análisis de materialidad como mínimo y un informe de control; también se genera otra nueva sección con un único indicador destinado a las entidades dependientes, aunque sólo exige la publicación del nombre de las fundaciones y entidades; un indicador destinado al porcentaje de profesores contratados que han leído su tesis en la propia universidad; y, por último, un indicador sobre la publicación del porcentaje de inserción laboral y de la mejora de empleabilidad. Al mismo tiempo, hay varios indicadores que refuerzan sus exigencias, aunque sin acoger a otros indicadores previos mediante la fusión de los mismos. Por citar algunos ejemplos: en la información sobre el claustro se exige que se identifique el sexo, los currículums de los profesores se deben publicar por departamento, o en los resultados se exige que también se publiquen los proyectos de investigación, la transferencia de conocimiento y las publicaciones JCR (Barrio et al., 2020; Barrio y Martin Cavanna, 2012). Estas modificaciones las podemos visualizar en la Tabla 3.

Tabla 3. Comparativa de indicadores utilizados por la Fundación Compromiso y Transparencia en sus informes de transparencia universitaria de 2011 y 2019

\begin{tabular}{|l|l|}
\hline \multicolumn{1}{|c|}{ Plan Estratégico } & \multicolumn{1}{c|}{ Plan Estratégico } \\
\hline $\begin{array}{l}\text { Publica las líneas generales de su plan } \\
\text { estratégico. }\end{array}$ & $\begin{array}{l}\text { Publica su misión y el plan estratégico y } \\
\text { el grado de cumplimiento. }\end{array}$ \\
\hline Misión & \\
\hline Publica su misión & Personal \\
\hline Personal & $\begin{array}{l}\text { Publica información sobre el personal } \\
\text { contratado: PAS y PDI. }\end{array}$ \\
\hline $\begin{array}{l}\text { Publica información sobre el personal } \\
\text { contratado: PAS y PDI. }\end{array}$ & $\begin{array}{l}\text { Publica información sobre bandas } \\
\text { salariales. }\end{array}$ \\
\hline $\begin{array}{l}\text { Publica información sobre bandas } \\
\text { salariales. }\end{array}$ & Políticas y Gobierno \\
\hline Gobierno & $\begin{array}{l}\text { Publica la composición de sus órganos } \\
\text { de gobierno, incluyendo organismos } \\
\text { unipersonales, mencionando nombres y } \\
\text { apellidos, titulación y responsabilidades. }\end{array}$ \\
\hline $\begin{array}{l}\text { Publica la composición de sus órganos } \\
\text { de gobierno, mencionando nombres y } \\
\text { apellidos, titulación y } \\
\text { responsabilidades. }\end{array}$ & \begin{tabular}{l} 
Publica sus estatutos y reglamentos. \\
\hline Publica sus estatutos.
\end{tabular} \\
\hline
\end{tabular}




\begin{tabular}{|c|c|}
\hline & $\begin{array}{l}\text { Publica las fechas de las reuniones y } \\
\text { acuerdos adoptados por sus órganos de } \\
\text { gobierno. }\end{array}$ \\
\hline Oferta y Demanda Académica & Oferta y Demanda Académica \\
\hline \multicolumn{2}{|l|}{$\begin{array}{l}\text { Publica su oferta de títulos reglados y } \\
\text { propios por ramas, campus y centros. }\end{array}$} \\
\hline $\begin{array}{l}\text { Publica el número de solicitudes de } \\
\text { matrícula demandadas en relación con } \\
\text { el número de plazas ofertadas }\end{array}$ & $\begin{array}{l}\text { Publica el número total de solicitudes, } \\
\text { admisiones y matrículas en relación con } \\
\text { el número total de las plazas ofertadas } \\
\text { por titulación }\end{array}$ \\
\hline $\begin{array}{l}\text { Publica el porcentaje y evolución de la } \\
\text { demanda de plazas }\end{array}$ & $\begin{array}{l}\text { Publica el porcentaje y evolución de la } \\
\text { demanda de plazas }\end{array}$ \\
\hline Claustro & Personal docente e investigador \\
\hline $\begin{array}{l}\text { Publica perfil y currículum de los } \\
\text { profesores por departamento o área } \\
\text { académica. }\end{array}$ & $\begin{array}{l}\text { Publica perfil y currículum de los } \\
\text { profesores por departamento o área } \\
\text { académica. }\end{array}$ \\
\hline \multirow[t]{2}{*}{$\begin{array}{l}\text { Publica el porcentaje de profesores } \\
\text { extranjeros de su claustro. }\end{array}$} & $\begin{array}{l}\text { Publica el porcentaje de profesores } \\
\text { extranjeros de su claustro. }\end{array}$ \\
\hline & $\begin{array}{l}\text { Publica el porcentaje de profesores } \\
\text { contratados que han leído la tesis en la } \\
\text { misma universidad que los contrata }\end{array}$ \\
\hline Alumnos & Alumnos \\
\hline \multicolumn{2}{|l|}{$\begin{array}{l}\text { Publica el número de alumnos } \\
\text { matriculados en cada uno de los títulos. }\end{array}$} \\
\hline $\begin{array}{l}\text { Facilita información sobre los } \\
\text { principales canales de representación y } \\
\text { comunicación con los alumnos. }\end{array}$ & $\begin{array}{l}\text { Facilita información sobre los principales } \\
\text { canales de representación y } \\
\text { comunicación con los alumnos. }\end{array}$ \\
\hline $\begin{array}{l}\text { Facilita información sobre la oferta de } \\
\text { becas y ayudas disponibles. }\end{array}$ & $\begin{array}{l}\text { Facilita información sobre la oferta de } \\
\text { becas y ayudas disponibles. }\end{array}$ \\
\hline Resultados & Resultados \\
\hline $\begin{array}{l}\text { Publica los principales resultados } \\
\text { relacionados con la labor investigadora } \\
\text { de sus profesores. }\end{array}$ & $\begin{array}{l}\text { Publica los principales resultados } \\
\text { relacionados con la labor investigadora } \\
\text { de sus profesores. }\end{array}$ \\
\hline $\begin{array}{l}\text { Publica los principales datos } \\
\text { relacionados con el rendimiento } \\
\text { académico de sus alumnos. }\end{array}$ & $\begin{array}{l}\text { Publica los principales datos } \\
\text { relacionados con el rendimiento } \\
\text { académico de sus alumnos. }\end{array}$ \\
\hline $\begin{array}{l}\text { Publica los índices de satisfacción de } \\
\text { los alumnos con los profesores, } \\
\text { servicios, oferta académica, etc. }\end{array}$ & $\begin{array}{l}\text { Publica los índices de satisfacción de los } \\
\text { alumnos con los profesores y los } \\
\text { servicios. }\end{array}$ \\
\hline $\begin{array}{l}\text { Publica su posición en los principales } \\
\text { rankings internacionales. }\end{array}$ & $\begin{array}{l}\text { Publica su posición en los principales } \\
\text { rankings nacionales y/o internacionales. }\end{array}$ \\
\hline
\end{tabular}


Publica el número y porcentaje de alumnos fuera de la comunidad y lo extranjeros matriculados en sus carreras.
Publica el número y porcentaje de alumnos fuera de la comunidad y los extranjeros matriculados en sus carreras y el número y porcentaje de alumnos en programas de movilidad cuyo propósito no sea titularse en España.

Facilitará información sobre el porcentaje de inserción laboral de sus graduados y/o la mejora de la empleabilidad de los que ya cuenten con empleo.

Información Económica Información Económica

Publica el presupuesto detallado y Publica su presupuesto aprobado y el grado de ejecución del mismo. aprobado correspondiente al año en curso.

\section{Publica los principales estados} financieros: Balance de Situación y Cuenta de Pérdidas y Ganancias.

Cuenta de Pérdidas y Ganancias.

Publica la memoria explicativa de las cuentas generales.

Publica el informe completo de la auditoría externa de sus cuentas.

Proporciona información desglosada de sus ingresos clasificados según sus diferentes fuentes.

Proporciona información desglosadas de sus gastos según su aplicación.
Publica la memoria explicativa de las cuentas generales.

Publica el informe completo de la auditoría externa de sus cuentas.

\section{Entidades dependientes}

Publica el nombre de sus fundaciones y entidades instrumentales dependientes

Consejo Social

Publica la dotación presupuestaria del consejo social y su estructura organizativa.

Publica un informe de responsabilidad social aprobado por el consejo social que deberá incluir un análisis de materialidad, cumplimiento de objetivos y metas.

Publica un informe de control, aprobado por el consejo social, en el que se incluya una breve descripción de las 
Fuente: elaboración propia a partir de la web de la Fundación Compromiso y Transparencia.

En cuanto a las debilidades o propuestas de mejora que podemos hacer a este índice de transparencia, hay varios ámbitos en los que podemos incidir. En primer lugar, y recordando los criterios generales que hemos identificado sobre las características de un índice de calidad y robustez evaluativa para la transparencia universitaria, hemos de hacer referencia a la calidad de la información. Este índice se limita a evaluar la cantidad de información publicada, aunque es cierto que exige un conjunto de informaciones de alta relevancia que sí permiten juzgar las acciones de la organización evaluada tanto a los miembros de la comunidad universitaria como al resto de la sociedad, deja de lado cuestiones importantes sobre cómo es esta información. En la edición de 2018 y 2019 se introduce un cambio que es consecuente con la exigencia de integridad de la información a la que hace referencia la fundación, algo que antes no sucedía como es la valoración gradual con 0 puntos para la no publicación, 1 punto para la publicación parcial y 2 puntos para la publicación total. Esto es, sin duda, un avance importante en la dirección correcta de evaluar la calidad de la información, ya que una de las características más elementales de dicha calidad es la exhaustividad de la información, no obstante, no sacia totalmente las exigencias de la misma.

Tomando de referencia a la Metodología de Evaluación y Seguimiento de la Transparencia de la Actividad Pública (MESTA en adelante), la primera metodología oficial para evaluar la transparencia pública en España, diseñada por el Consejo de la Transparencia y Buen Gobierno (CTBG en adelante), hay dos criterios altamente relevantes que la metodología de la fundación no tiene en cuenta: la claridad del lenguaje y la apertura del formato de los datos, es decir, que estos sean reutilizables. Estos dos criterios no son tenidos en cuenta en ningún caso en esta metodología, pero sabemos que la claridad del lenguaje es básica para que la información pueda ser comprensible por toda la sociedad, y al mismo tiempo, que la oportunidad de reutilizar los datos hace que la información pueda ser manejada, procesada y entendida mucho mejor en determinados casos. Con una información con un lenguaje complejo y tecnicista en formato de documento portable (PDF por sus siglas en inglés) no procesable, es evidente que se generará una peor comprensión, incluso aunque los 
datos publicados sean los mismos. Por tanto, estos dos criterios son de alta relevancia y deben ser tenidos en cuenta.

Al mismo tiempo, hay otros criterios que son mencionados en esta metodología, pero que no queda claro cómo se evalúan o si se evalúan en algún caso. Nos referimos a la visibilidad de la información, a la actualización y a la accesibilidad. Entendemos que, al introducir el criterio de la gradualidad en la valoración con notas de 0 a 2 puntos, tanto la actualización de la información como su integridad, de la que antes hemos hablado, será valorada al mismo tiempo que la publicación de la información, es decir, que sólo cuando la información es completa y esté actualizada se valorará con 2 puntos. Sin embargo, no queda claro en absoluto cómo se valora la visibilidad de la información y la accesibilidad web, ya que no hay ningún indicador específico para ello ni es una característica de la información contenida en cada indicador, sino características del continente de la información, la página web. Por tanto, esta debilidad de la metodología de la fundación puede corregirse, al igual que hacen otras metodologías, generando un apartado aparte para evaluar las características de la web. En este apartado, proponemos que se incluyan cuatro indicadores: la existencia de un lugar específico de transparencia, la visibilidad de la información dentro de la página web, la unificación o diseminación de la información y la accesibilidad web (criterios bastante parecidos a los empleados por MESTA para evaluar el soporte web). Sin embargo, la accesibilidad web, aunque deseable es de muy difícil aplicación, ya que sería necesario mecanismos de evaluación uniformes por países que pudieran generar exámenes de dicha accesibilidad para las distintas webs de las universidades latinoamericanas.

Figura 1. Evolución de la transparencia de las universidades públicas españolas según los informes de la Fundación Compromiso y Transparencia



Fuente: Elaboración propia a partir de Barrio et al. (2020). 
En cuanto a los indicadores, consideramos que los empleados por la fundación son de una alta conveniencia y, además, han sido testados a lo largo de la experiencia de aplicación de la metodología durante nueve ediciones, lo que les confiere una oportunidad demostrada. No obstante, conviene realizar una serie de salvedades. En primer lugar, es preciso reflexionar sobre la evolución de los propios indicadores: la realidad universitaria española ha evolucionado notablemente en los últimos años, también en cuanto a transparencia se refiere, no en vano, como muestra la Figura 1, sacado del informe de 2019 de la Fundación Compromiso y Transparencia, las universidades opacas han pasado de ser las más numerosas a las menos y el caso contrario ha sucedido con las transparentes. Esto indica una evolución muy positiva que conecta tanto con un proceso social de apertura y progreso organizativo, como con un proceso de aprendizaje de las instituciones evaluadas que van incorporando progresivamente los indicadores demandados por un ranking de referencia, con considerable impacto académico y público y sostenido en el tiempo. Sin embargo, esto no es igualmente esperable en el contexto latinoamericano en el que, como ya señalamos, sólo se han producido evaluaciones aisladas y no sostenidas en el tiempo, la mayoría de las veces con escaso impacto en la opinión pública. Por tanto, no deberían desecharse los indicadores que con el paso del tiempo la fundación ha ido desplazando por cumplirse en la mayoría de los casos. Estos indicadores son sólo dos: la oferta de títulos reglados y propios por ramas, campus y centros y el número de alumnos matriculados en cada título o grado ofertado.

En cuanto al proceso de evaluación, la Fundación Compromiso y Transparencia no aclara en sus informes cuál es la secuencia que sigue para llevar a cabo los mismos. No obstante, en la mayoría de casos como puede ser la evaluación con MESTA o las ediciones realizadas por Transparencia Internacional España (CTBG, 2017; Ros Medina, 2018), se suele realizar la evaluación en contacto con el sujeto evaluado, es decir, que se contacta con el mismo para, o bien solicitarle que sea él el que realice una primera evaluación de los contenidos que publica y aporte enlaces webs a los mismos para que posteriormente sean verificados por la entidad evaluadora, o bien se le remite una evaluación inicial para que pueda ser revisada por el sujeto evaluado y discutida en su caso aportando nueva información. Es más, durante estos procesos se suele aceptar que la información que es incorporada durante los mismos es tenida en cuenta de forma positiva. Este diseño evaluativo colaborativo también es un punto a favor para que las entidades progresen a lo largo del tiempo, ya que van comprobando de primera mano sus carencias y las posibles mejoras que podrían aplicar. Por ello, creemos que lo más útil para poner en marcha un nuevo índice de evaluación de la transparencia de las universidades en América Latina es realizar un diseño colaborativo, optando por el envío 
a los sujetos obligados de una primera evaluación tentativa, para que sea revisada por los mismos con un plazo de tiempo suficiente que permita la publicación de nuevos contenidos, para posteriormente remitir resultados y programar una reunión final entre evaluados y evaluadores que ponga en común carencias y estrategias de mejora.

\section{La transparencia activa de la Universidad de Buenos Aires, como estudio de caso piloto para la puesta en marcha de un Índice de Transparencia de las Universidades Latinoamericanas (INTULA)}

En este caso de estudio piloto hemos elegido la Universidad de Buenos Aires (UBA en adelante), una universidad que cumple sus 200 años de historia el 12 de agosto de 2021, siendo una de las universidades históricamente más prestigiosas de América Latina. En el Ranking QS Global lleva 7 años consecutivos siendo la universidad más altamente valorada de toda Iberoamérica, teniendo en cuenta también España, apareciendo concretamente en la edición de 2022 en el puesto 69 del mundo. Es una institución que cuenta, además, con más de 290.000 estudiantes, según estadísticas de 2016, siendo de largo la universidad con más alumnos de Argentina, concentrado en torno al $14 \%$ de los estudiantes de educación terciaria del país según datos del propio Ministerio de Educación de Argentina, una de las universidades con más alumnos del mundo y la segunda de Iberoamérica, sólo por detrás de la Universidad Nacional Autónoma de México, que cuenta con más de 366.000 estudiantes en $\underline{2021}$. Además, según sus estadísticas, en 2011 concentraba a más de 28.000 docentes entre grado y postgrado, de los que llama la atención que más del $23 \%$ en dicho curso lo fueron ad honorem. Por último, decir que cuenta con 13 facultades y 61 institutos de investigación, 44 de ellos en alianza con el Consejo Nacional de Investigaciones Científicas y Técnicas (CONICET).

Hemos elegido esta institución para hacer frente a una evaluación piloto por ser una de las universidades más importantes en todos los parámetros académicos, sociales y de investigación de Latinoamérica, lo que también nos lleva a pensar que la transparencia de la dicha institución puede ser mayor que la de otros centros universitarios que cuenten con menos arraigo y éxito en estos parámetros. Optamos, aunque defendemos que el diseño de una evaluación de este tipo debe ser colaborativo, por realizar este estudio piloto sin la participación de las autoridades de la Universidad de Buenos Aires, por cuanto ello habría convertido dicha prueba en mucho más costosa y difícil de realizar a nivel temporal y humano. Por tanto, sometemos a un análisis tentativo de los indicadores manejados por la Fundación Compromiso y Transparencia 
para su evaluación de la transparencia universitaria en España, con el añadido de que mantenemos sus dos indicadores eliminados desde su primera edición (publicación de la oferta educativa y del número de alumnos por titulaciones), introducimos una valoración sobre el lenguaje fácil y la reutilización de la información y, por último, añadimos una nueva sección de evaluación de la transparencia del soporte web, en consonancia con los criterios empleados en MESTA.

De esta forma, ofrecemos tres valoraciones distintas, como sigue a continuación: 1) una valoración empleando los mismos criterios e indicadores que la Fundación Compromiso y Transparencia, que es comparable directamente con las valoraciones realizadas por dicho organismo para las universidades españolas, 2) una valoración que emplea como base dicha metodología pero añade los dos indicadores eliminados de su primera edición, así como los criterios reforzados de calidad sobre lenguaje fácil y reutilización de los datos, 3) y, por último, la que incluye todas las recomendaciones de mejora realizadas durante este trabajo a la metodología de la fundación, a saber: los dos indicadores eliminados desde su primera edición, los criterios de calidad de la información sobre lenguaje fácil y apertura de datos, así como un apartado de transparencia del soporte web. Esta última, es la propuesta que consideramos que debería aplicarse a Latinoamérica en un índice transnacional que someta a examen a las universidades más importantes del continente. El soporte web tendrá una valoración del $20 \%$, mientras que el $80 \%$ restante corresponderá a la valoración de la cantidad y calidad de la información clave publicada.

Tabla 5. Resultados de la Evaluación de la Transparencia de la Universidad de Buenos Aires

\begin{tabular}{|l|c|c|c|}
\cline { 2 - 4 } \multicolumn{1}{c|}{} & Nota 1 & Nota 2 & Nota 3 \\
\hline Sobre 2 & 0,59 & 0,42 & 0,67 \\
\hline Sobre 10 & 2,95 & 2,11 & 3,36 \\
\hline Sobre 100 & 29,50 & 21,12 & 33,56 \\
\hline
\end{tabular}

En cuanto a los resultados concretos de la evaluación de la transparencia de la UBA, han sido muy decepcionantes. En la nota 1, que es comparable con la de la que realiza la Fundación Compromiso y Transparencia en España, la UBA ha obtenido un 3,15 sobre 10 y ha cumplido sólo 4 criterios completamente y 9 criterios parcialmente, obteniendo una puntuación de 17 puntos, lo que le confiere sobradamente la categoría de "opaca" según la definición de la fundación, que fija en esta categoría a las 
universidades públicas que obtienen menos de 30 puntos; en la de "traslúcida" a las que obtienen de 30 a 39 puntos y como "transparentes" a aquellas que obtienen más de 40 y además cumplen con los indicadores de transparencia financiera. La UBA no sólo no publica la mayoría de los indicadores sometidos a estudio, sino que, además, prácticamente no publica información financiera, algo que es vital para permitir una efectiva rendición de cuentas. La nota 2 , que incluye el criterio reforzado de lenguaje comprensible y la exigencia de formatos abiertos, no cambia sustancialmente, pero sí se reduce hasta el 2,25 sobre 10 y los 16 puntos. En cambio, la nota 3 es la más elevada, con un 3,47 , debido a que la UBA obtiene una alta valoración en la transparencia de su soporte web, aunque seguiría siendo un suspenso bajo.

A nivel concreto, destacamos la opacidad reinante en el apartado de personal docente e investigador, en los resultados y en la información financiera, tres de las ramas de transparencia más altamente importantes para poder juzgar las acciones de la institución y los resultados de las mismas. Sin esta información, no es posible que se produzca rendición de cuentas al ser áreas elementales. Caso contrario son las áreas de política y gobierno, personal, alumnos, y, en menor medida, la de oferta y demanda académica y entidades dependientes. Caso aparte merece el apartado de consejo social o consejo consultivo, un órgano que es generalizado en el marco de las universidades públicas españolas; la UBA no parece tenerlo a escala global, aunque sí hay consejos consultivos menores por facultades y por departamentos, pero no hemos encontrado información como la demandada en los indicadores sobre presupuestos, informes de materialidad e informes de control.

\section{CONCLUSIONES.}

En este trabajo hemos puesto de relevancia la importancia que tiene la transparencia dentro del paradigma organizativo de la apertura y el gobierno abierto para convertir a las universidades públicas y privadas de Latinoamérica en organizaciones que sigan liderando el cambio y el desarrollo social en la época post-covid. Los retos a los que las sociedades latinoamericanas se habrán de enfrentar, fruto de sus contextos preexistentes, en esta nueva época serán mayores incluso que los de otros lugares como Europa o Norteamérica. Las universidades deben, por tanto, realizar un esfuerzo de apertura y transparencia tanto interna como hacia el exterior.

Sin embargo, la experiencia evaluativa de la transparencia universitaria ha sido menor y no se ha sostenido en el tiempo en esta región. Tampoco se ha producido una evaluación transversal de las universidades más relevantes del continente. Por ello, es momento de poner en práctica un índice internacional que aprenda de experiencias consolidadas en este ámbito como la puesta en marcha durante nueve ediciones hasta 
la fecha por la Fundación Compromiso y Transparencia en España para las universidades públicas y privadas. Esta metodología ha asentado el uso de 27 indicadores claves para juzgar el accionar de las universidades, así como sus resultados, juzgando la integridad y actualización de la información.

En este artículo hemos aplicado dicha metodología enriquecida con nuestras propuestas de mejora metodológicas, a saber: evaluar la comprensibilidad del lenguaje, el formato de los datos dando preferencia a los datos abiertos y las características de transparencia y apertura del formato web. De esta forma, hemos sometido a análisis la web de la Universidad de Buenos Aires, que es de largo la mejor universidad iberoamericana según el QS Ranking desde hace casi una década. Sin embargo, los resultados de dicha evaluación han sido ampliamente decepcionantes. La UBA ha cumplido sólo 17 puntos de 54 según la metodología de la Fundación Compromiso y

Transparencia, lo que le confiere sobradamente el calificativo de "opaca" según la clasificación usada por esta entidad. Además, esta nota vendría a suponer un 3,15 sobre 10 en transparencia, suspenso que aún se reduciría más si introducimos nuestros criterios reforzados de calidad de la información valorando facilidad del lenguaje y formato abierto de los datos, hasta el 2,25 sobre 10. En cambio, teniendo en cuenta junto a esta nota, la valoración sobre el soporte web de la UBA (siendo el $80 \%$ la nota sobre contenido y $20 \%$ la nota sobre continente), se incrementaría la misma hasta el 3,47 , lo que sigue siendo un suspenso bajo.

En definitiva, esta experiencia piloto nos revela que hasta una de las universidades más relevantes del continente por resultados académicos, población estudiantil e impacto social es una universidad opaca que está muy lejos del ideal de transparencia y apertura que sería deseable y, probablemente, exigible para enfrentar esta nueva y difícil situación post-covid. Por ello, se hace más necesario todavía la puesta en marcha de un ranking de referencia que evalúe la transparencia de las universidades públicas y privadas más importantes del continente, para colaborar efectivamente en hacer un diagnóstico fiable de la situación y proponer e incentivar acciones claras de mejora.

\section{REFERENCIAS BIBLIOGRÁFICAS}

Barrio, E., Martin Cavanna, J., 2012. Examen de transparencia. Informe de transparencia en la web de las universidades españolas en 2011. Madrid, España: Fundación Compromiso y Transparencia.

Barrio, E., Martin Cavanna, J., Sacristán Sánchez, C. (2020). Examen de transparencia. Informe de transparencia en la web de las universidades españolas en 2019. 
Madrid, España: Fundación Compromiso y Transparencia.

Cousido, P., Said-Hung, E., Montoya, C. (2017). La transparencia de las Universidades en Colombia en Internet. Transinformaçao, 29, 257-265.

CTBG, 2017. Informe de evaluación de los órganos constitucionales y reguladores. Madrid.

Kaufmann, D., Kraay, A., Zoido-lobatón, P. (1999). Governance Matters. World Bank Work. Pap. 2196, 1-68.

Mamani Bravo, W.J., Medina Cárdenas, A.D., Sáenz Rodríguez, J.E., Valencia Bellido, C.N. (2019). Transparencia Corporativa en las Universidades Privadas del Perú. Lima, Perú: Pontificia Universidad Católica del Perú.

O’Donnell, G. (1994). Delegative democracy. Journal of Democracy, 5, 55-69.

Parra Flórez, J.M. (2018). La transparencia y el gobierno corporativo: Un análisis a las universidades ubicadas en Centro América, en: Tovar-Gálvez, J.C. (Ed.), Trends and Challenges in Higher Education in Latin America, 116-129, Eindhoven, Paises Bajos: Adaya Press,.

Ros Medina, J.L. (2018). La evaluación de la transparencia en España a debate metodológico: MESTA e índices de Transparencia Internacional. Rev. Int. Transparencia e Integridad, 6.

Rothstein, B., Teorell, J. (2008). What is Quality of Government? A Theory of Impartial Institutions. Governance, 21, 165-190.

Zorzal, L. (2015). Transparência das informações das universidades federais: estudo dos relatórios de gestão à luz dos princípios de boa governança na administração pública federal. Brasilia, Brasil: Universidad de Brasilia. 


\section{ANEXOS}

Tabla 6. Formulario de Evaluación aplicado a la Universidad de Buenos Aires

\begin{tabular}{|c|c|c|c|c|c|c|}
\hline & Publicación & Actualización & $\begin{array}{l}\text { Lenguaje } \\
\text { fácil }\end{array}$ & Reutilización & $\begin{array}{c}\text { Nota } \\
1\end{array}$ & $\begin{array}{c}\text { Nota } \\
2\end{array}$ \\
\hline \multicolumn{7}{|l|}{ Plan Estratégico } \\
\hline $\begin{array}{l}\text { Publica su misión y el } \\
\text { plan estratégico y el } \\
\text { grado de cumplimiento. }\end{array}$ & 0 & 0 & 0 & 0 & 0 & 0 \\
\hline \multicolumn{7}{|l|}{ Personal } \\
\hline $\begin{array}{l}\text { Publica información } \\
\text { sobre el personal } \\
\text { contratado: PAS y PDI. }\end{array}$ & 2 & 0 & 0 & 0 & 1 & 1 \\
\hline $\begin{array}{l}\text { Publica información } \\
\text { sobre bandas salariales. }\end{array}$ & 2 & 1 & 1 & 0 & 1 & 1 \\
\hline \multicolumn{7}{|l|}{ Políticas y Gobierno } \\
\hline $\begin{array}{l}\text { Publica la composición } \\
\text { de sus órganos de } \\
\text { gobierno, incluyendo } \\
\text { organismos } \\
\text { unipersonales, } \\
\text { mencionando nombres y } \\
\text { apellidos, titulación y } \\
\text { responsabilidades. }\end{array}$ & 2 & 2 & 1 & 1 & 2 & 1,5 \\
\hline $\begin{array}{l}\text { Publica sus estatutos y } \\
\text { reglamentos. }\end{array}$ & 2 & 2 & 0 & 2 & 2 & 1,5 \\
\hline $\begin{array}{l}\text { Publica las fechas de las } \\
\text { reuniones y acuerdos } \\
\text { adoptados por sus } \\
\text { órganos de gobierno. }\end{array}$ & 2 & 2 & 0 & 1 & 2 & 1,25 \\
\hline \multicolumn{7}{|l|}{$\begin{array}{l}\text { Oferta y Demanda } \\
\text { Académica }\end{array}$} \\
\hline $\begin{array}{l}\text { Publica el número total } \\
\text { de solicitudes, } \\
\text { admisiones y matrículas } \\
\text { en relación con el } \\
\text { número total de las } \\
\text { plazas ofertadas por } \\
\text { titulación }\end{array}$ & 1 & 0 & 1 & 2 & 1 & 1 \\
\hline $\begin{array}{l}\text { Publica el porcentaje y } \\
\text { evolución de la } \\
\text { demanda de plazas }\end{array}$ & 0 & 0 & 0 & 0 & 0 & 0 \\
\hline
\end{tabular}




\begin{tabular}{|c|c|c|c|c|c|c|}
\hline \multicolumn{5}{|l|}{$\begin{array}{l}\text { Personal docente e } \\
\text { investigador }\end{array}$} & \multirow[b]{2}{*}{1} & \multirow{2}{*}{0,75} \\
\hline $\begin{array}{l}\text { Publica perfil y } \\
\text { currículum de los } \\
\text { profesores por } \\
\text { departamento o área } \\
\text { académica. }\end{array}$ & 1 & 1 & 0 & 1 & & \\
\hline $\begin{array}{l}\text { Publica el porcentaje de } \\
\text { profesores extranjeros } \\
\text { de su claustro. }\end{array}$ & 0 & 0 & 0 & 0 & 0 & 0 \\
\hline $\begin{array}{l}\text { Publica el porcentaje de } \\
\text { profesores contratados } \\
\text { que han leído la tesis en } \\
\text { la misma universidad } \\
\text { que los contrata }\end{array}$ & 0 & 0 & 0 & 0 & 0 & 0 \\
\hline \multicolumn{7}{|l|}{ Alumnos } \\
\hline $\begin{array}{l}\text { Facilita información } \\
\text { sobre los principales } \\
\text { canales de } \\
\text { representación y } \\
\text { comunicación con los } \\
\text { alumnos. }\end{array}$ & 2 & 1 & 1 & 1 & 1 & 1,25 \\
\hline $\begin{array}{l}\text { Facilita información } \\
\text { sobre la oferta de becas } \\
\text { y ayudas disponibles. }\end{array}$ & 2 & 2 & 1 & 1 & 2 & 1,5 \\
\hline \multicolumn{7}{|l|}{ Resultados } \\
\hline $\begin{array}{l}\text { Publica los principales } \\
\text { resultados relacionados } \\
\text { con la labor } \\
\text { investigadora de sus } \\
\text { profesores. }\end{array}$ & 0 & 0 & 0 & 0 & 0 & 0 \\
\hline $\begin{array}{l}\text { Publica los principales } \\
\text { datos relacionados con } \\
\text { el rendimiento } \\
\text { académico de sus } \\
\text { alumnos. }\end{array}$ & 0 & 0 & 0 & 0 & 0 & 0 \\
\hline $\begin{array}{l}\text { Publica los índices de } \\
\text { satisfacción de los } \\
\text { alumnos con los } \\
\text { profesores y los } \\
\text { servicios. }\end{array}$ & 0 & 0 & 0 & 0 & 0 & 0 \\
\hline $\begin{array}{l}\text { Publica su posición en } \\
\text { los principales rankings } \\
\text { nacionales y/o } \\
\text { internacionales. }\end{array}$ & 1 & 2 & 1 & 0 & 1 & 1 \\
\hline
\end{tabular}




\begin{tabular}{|c|c|c|c|c|c|c|}
\hline $\begin{array}{l}\text { Publica el número y } \\
\text { porcentaje de alumnos } \\
\text { fuera de la comunidad y } \\
\text { los extranjeros } \\
\text { matriculados en sus } \\
\text { carreras y el número y } \\
\text { porcentaje de alumnos } \\
\text { en programas de } \\
\text { movilidad cuyo propósito } \\
\text { no sea titularse en } \\
\text { España. }\end{array}$ & 0 & 0 & 0 & 0 & 0 & 0 \\
\hline $\begin{array}{l}\text { Facilitará información } \\
\text { sobre el porcentaje de } \\
\text { inserción laboral de sus } \\
\text { graduados y/o la mejora } \\
\text { de la empleabilidad de } \\
\text { los que ya cuenten con } \\
\text { empleo. }\end{array}$ & 0 & 0 & 0 & 0 & 0 & 0 \\
\hline \multicolumn{7}{|l|}{$\begin{array}{l}\text { Información } \\
\text { Económica }\end{array}$} \\
\hline $\begin{array}{l}\text { Publica el presupuesto } \\
\text { detallado y aprobado } \\
\text { correspondiente al año } \\
\text { en curso. }\end{array}$ & 1 & 0 & 0 & 0 & 1 & 0,25 \\
\hline $\begin{array}{l}\text { Publica los principales } \\
\text { estados financieros: } \\
\text { Balance de Situación y } \\
\text { Cuenta de Pérdidas y } \\
\text { Ganancias. }\end{array}$ & 0 & 0 & 0 & 0 & 0 & 0 \\
\hline $\begin{array}{l}\text { Publica la memoria } \\
\text { explicativa de las } \\
\text { cuentas generales. }\end{array}$ & 0 & 0 & 0 & 0 & 0 & 0 \\
\hline $\begin{array}{l}\text { Publica el informe } \\
\text { completo de la auditoría } \\
\text { externa de sus cuentas. }\end{array}$ & 2 & 0 & 0 & 0 & 1 & 0,5 \\
\hline \multicolumn{7}{|l|}{$\begin{array}{l}\text { Entidades } \\
\text { dependientes }\end{array}$} \\
\hline $\begin{array}{l}\text { Publica el nombre de } \\
\text { sus fundaciones y } \\
\text { entidades } \\
\text { instrumentales } \\
\text { dependientes }\end{array}$ & 1 & 1 & 0 & 0 & 1 & 0,5 \\
\hline \multicolumn{7}{|l|}{ Consejo Social } \\
\hline $\begin{array}{l}\text { Publica la dotación } \\
\text { presupuestaria del } \\
\text { consejo social y su } \\
\text { estructura organizativa. }\end{array}$ & 0 & 0 & 0 & 0 & 0 & 0 \\
\hline
\end{tabular}



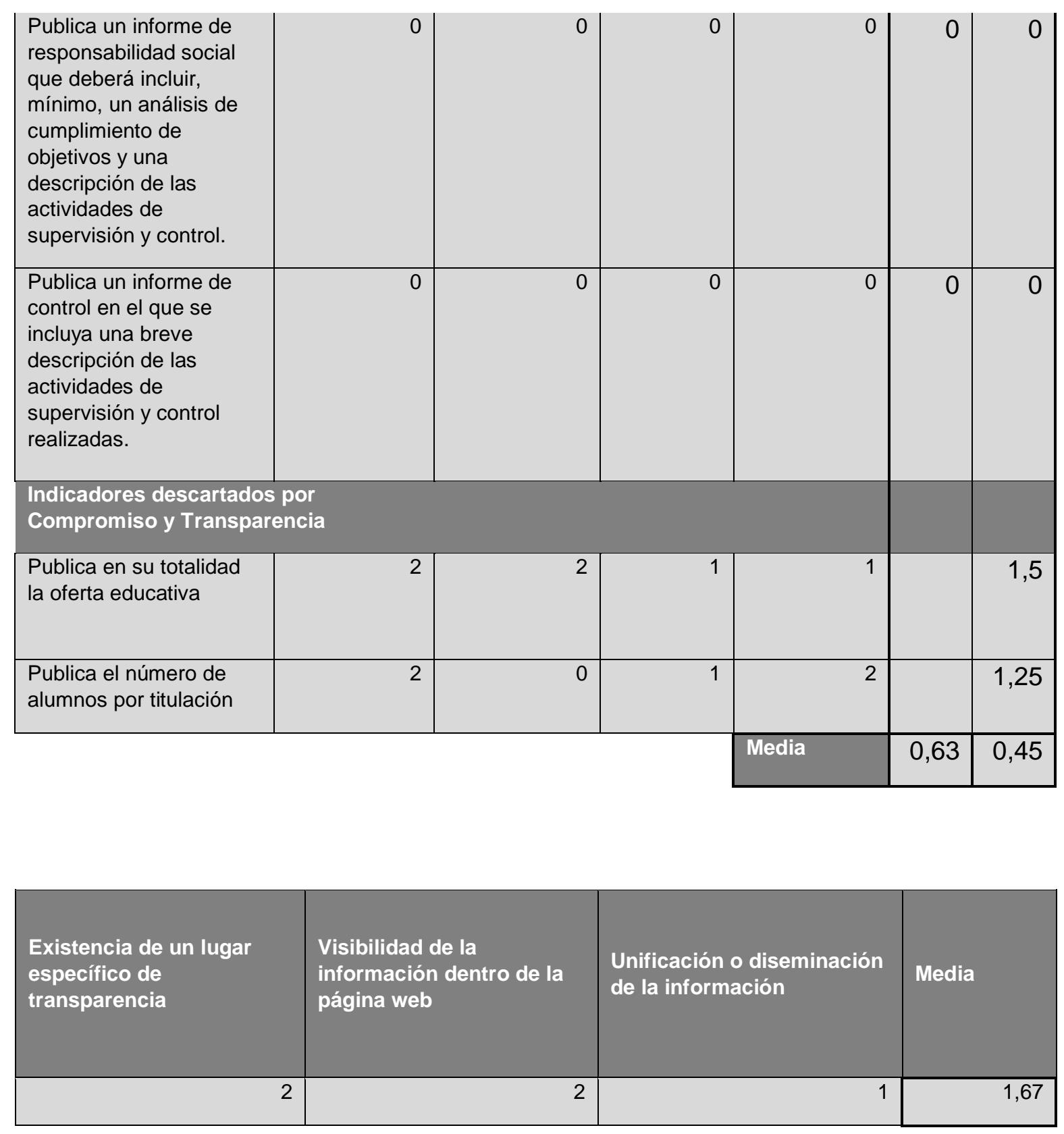
Autor(s): Ros-Medica José Luis, ORCID: 0000-0003-2903-6632

Mañogil Ros Alejandro, correo: alejandro.manogilr@um.es

Figura 2. Representación gráfica de los resultados del INTULA para la UBA

\section{INTULA - UBA}

1 NOTA 1

Valoración empleando los mismos criterios e indicadores que Compromiso y

Transparencia (CyT), facilitando su

comparación con las valoraciones

realizadas para universidades españolas

2 NOTA 2

Valoración empleando como base la metodología de CyT, pero añadiendo los dos indicadores eliminados de su primera edición y criterios reforzados sobre lenguaje fácil y reutilización de los datos

\section{NOTA 3}

Metodología de СуT, añadiendo: los dos indicadores eliminados desde su primera edición, los criterios de calidad sobre lenguaje fácil y apertura de datos y un apartado de transparencia del soporte web

Índice de Transparencia Universitaria en Latino América Universidad de Buenos Aires Proyecto Piloto
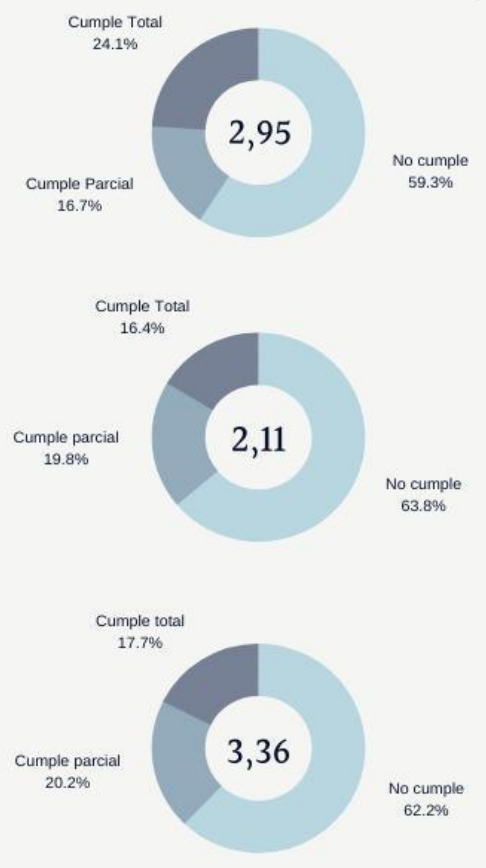